\title{
OBSERVATIONAL CONSTRAINTS ON MODEL ATMOSPHERES FOR URANUS AND NEPTUNE
}

\author{
THÉRÈSE ENCRENAZ*, JOHANNES HARDORP, and TOBIAS OWEN** \\ Dept. of Earth and Space Sciences, State University of New York at Stony Brook, \\ Stony Brook, N.Y., U.S.A. \\ and \\ JERRY H. WOODMAN \\ McDonald Observatory, University of Texas, Fort Davis, Tex., U.S.A.
}

\begin{abstract}
We have re-examined the visible and near-IR regions of the spectra of Uranus and Neptune to provide additional data for constructing atmospheric models. We find that a true continuum exists only at wavelengths below $4700 \AA$, that the $6420 \AA$ absorption previously attributed to hydrogen is probably caused by methane, that there is no evidence for ammonia, ethylene or ethane absorptions in our spectra, and that the abundance of methane is probably much higher than previous estimates suggest. This last finding implies that the value of $\mathrm{H} / \mathrm{C}$ in the atmospheres of both planets is much less than $1 / 10$ the solar (or Jovian) value. Clear, Rayleigh-scattering model atmospheres are not compatible with the observations, but more work is needed to establish viable alternatives.
\end{abstract}

\section{Introduction}

There has been considerable interest in recent years in the structure and composition of the atmospheres of Uranus and Neptune. These distant planets are not easy objects to study at the telescope, so efforts to understand their atmospheres have been severely hampered by a lack of useful observational data. In the present paper, we first review some current ideas about both planets and then offer some new data which should be incorporated in future atmospheric models.

Belton, McElroy, and Price (1971 - hereinafter referred to as BMP) have suggested that the atmospheres of both of these planets are devoid of clouds in the regions accessible to optical observations. This means that the transfer of radiation outside molecular absorption bands proceeds by Rayleigh and Raman scattering in the semi-infinite, pure molecular atmospheres. A further refinement to the original model was offered by Wallace (1972) who considered the effects of Raman scattering in greater detail.

This model has been challenged by several authors. Sinton (1972) and Westphal (1972) both reported observations of limb brightening on the disk of the planet when it was viewed in the region of a strong methane absorption band. Sinton (1972) also found brightening over the south pole of the planet. By analogy with similar observations of Jupiter (Owen, 1969), both Sinton and Westphal concluded that their results supported a model in which a high cloud layer was overlain by a layer of absorbing and scattering gas. Belton and Price (1973) have responded that limb brightening could be expected in strong methane bands in a pure molecular model as well.

* Present address: Groupe Planètes, Observatoire de Meudon, Meudon, France.

** Guest investigator, Hale Observatories, 1972. 
However, their discussion cannot account for the bright features on the disk itself that are apparent in Sinton's (1972) photograph.

Prinn and Lewis (1973) have presented thermodynamic arguments for the presence of a high methane haze in the atmosphere of Uranus. Danielson et al. (1972) found that limb darkening of Uranus in the visible and near UV could not be matched by a semi-infinite atmosphere, but could be explained by a model incorporating a cloud layer beneath a layer of gas. Belton et al. (1973) have found evidence for Raman scattering in the atmosphere of Uranus, but they argue that this evidence does not really permit a conclusive discrimination among various models. In a new discussion of the pressure-induced absorptions of hydrogen, Belton and Spinrad (1973) concluded that the semi-infinite model does not fit the observations as well as a model with a cloud layer. Finally, Savage and Caldwell (1973) have presented the results of OAO short wavelength photometry which again do not agree with the predictions for a semiinfinite atmosphere: the planets are not as bright at short wavelengths as would be expected in the absence of clouds or haze.

In short, the weight of presently available evidence appears to be against the model originally proposed by BMP. We now wish to present some additional new data, including our own, which should be incorporated in attempts to develop more sophisticated models in the future.

\section{Density and Bulk Composition}

New determinations of the radii of both planets have suggested that they are larger than had been thought. This means the densities are lower, as shown below:

\begin{tabular}{lccc} 
& $M$ & $R$ & $Q$ \\
\hline Uranus & 15 & 4.04 & 1.2 \\
Neptune & 17 & 3.87 & 1.7
\end{tabular}

Masses and radii are given in terms of terrestrial values; the new radii are taken from Danielson et al. (1972) - Uranus, and Kovalevsky and Link (1969) - Neptune.

While these densities are lower than the 'textbook' values of 1.60 and 2.25 (Allen, 1963), they still do not permit the planets to be composed primarily of hydrogen, as is the case for Jupiter and Saturn (Smoluchowski, 1973). The precision of the present data is probably still too poor to exclude the possibility that the bulk composition of the two planets is identical.

\section{The Radio and Thermal IR Spectra}

A useful summary of available observations from $10 \mu$ to $10 \mathrm{~cm}$ has been given by Morrison and Cruikshank (1973), who pointed out the essential similarity of the 
thermal spectrum from each planet. These authors used this similarity to suggest that if the chemical composition of the two planets is also the same, 'it appears that they have essentially the same atmospheric structure in spite of their differences in distances from the Sun and in inclination of their axes of rotation'. We shall return to this point since it has an obvious bearing on any attempts to develop model atmospheres.

A recent observation of Uranus at $21 \mathrm{~cm}$ by Briggs (1973) revealed a temperature of $280 \pm 60 \mathrm{~K}$ at this wavelength, suggesting that these planets, like Jupiter and Saturn, probably have regions in their lower atmospheres that are well above the freezing point of water.

\section{The Near-IR Continuum}

BMP suggested that the depressed near-IR continuum of the spectra of both planets could be explained by the far wing of the pressure-induced fundamental of $\mathrm{H}_{2}$. This discussion ignored the effects of weak methane bands. In reality, it is virtually impossible to find a region in this part of the spectrum of either planet where weak methane lines are not present. The contrary impression exists simply because of observations made at low spectral resolution.

An excellent example of this is the $7500 \AA$ region. At very low resolution, this appears to be a 'window' in the planetary spectra, but as the resolution is increased, more and more lines appear (Kuiper, 1949; Owen, 1967; Lutz and Ramsay, 1972). These lines are all methane, and one may predict with reasonable certainty that still higher resolution would probably add to their number.

Another place where this effect occurs is at $6400 \AA$. Scanner observations unaffected by the vagaries of photographic emulsions clearly show that the continuum in this region is badly distorted. Once again, high resolution coupled with appropriate laboratory data indicates that weak methane lines are present. It is our current assessment that an undistorted continuum exists only at wavelengths below $4700 \AA$ (see below).

\section{The $6420 \AA$ Region and the $4-0 \mathrm{H}_{2}$ Dipole Absorptions}

Spinrad (1963) felt that he had identified the $S_{4}(0)$ pressure-induced absorption from $\mathrm{H}_{2}$ in the spectra of both Uranus and Neptune at $6420 \AA$. A careful study of the problem by Belton and Spinrad (1973) has led to the conclusion that this identification was probably incorrect because of the absence of the $S_{4}(1)$ line in the planetary spectra. It would occur at $6370 \AA$, in a region that seems relatively free of methane absorption.

We have confirmed the absence of the $S_{4}(1)$ line and we feel we have been able to show that the $6420 \AA$ absorption is in fact caused by methane (Figure 1 - Owen et al., 1974). This gas also appears to be responsible for an absorption at $6490 \AA$ reported by Spinrad (1963) and confirmed by Teifel and Kharitonova (1970). This at present there seems to be no reason to assume that the $6420 \AA$ feature is caused by $\mathrm{H}_{2}$, although this gas make some contribution to the observed absorption. Abundances of $\mathrm{H}_{2}$ based interpretations of this feature are therefore unlikely to be correct. 


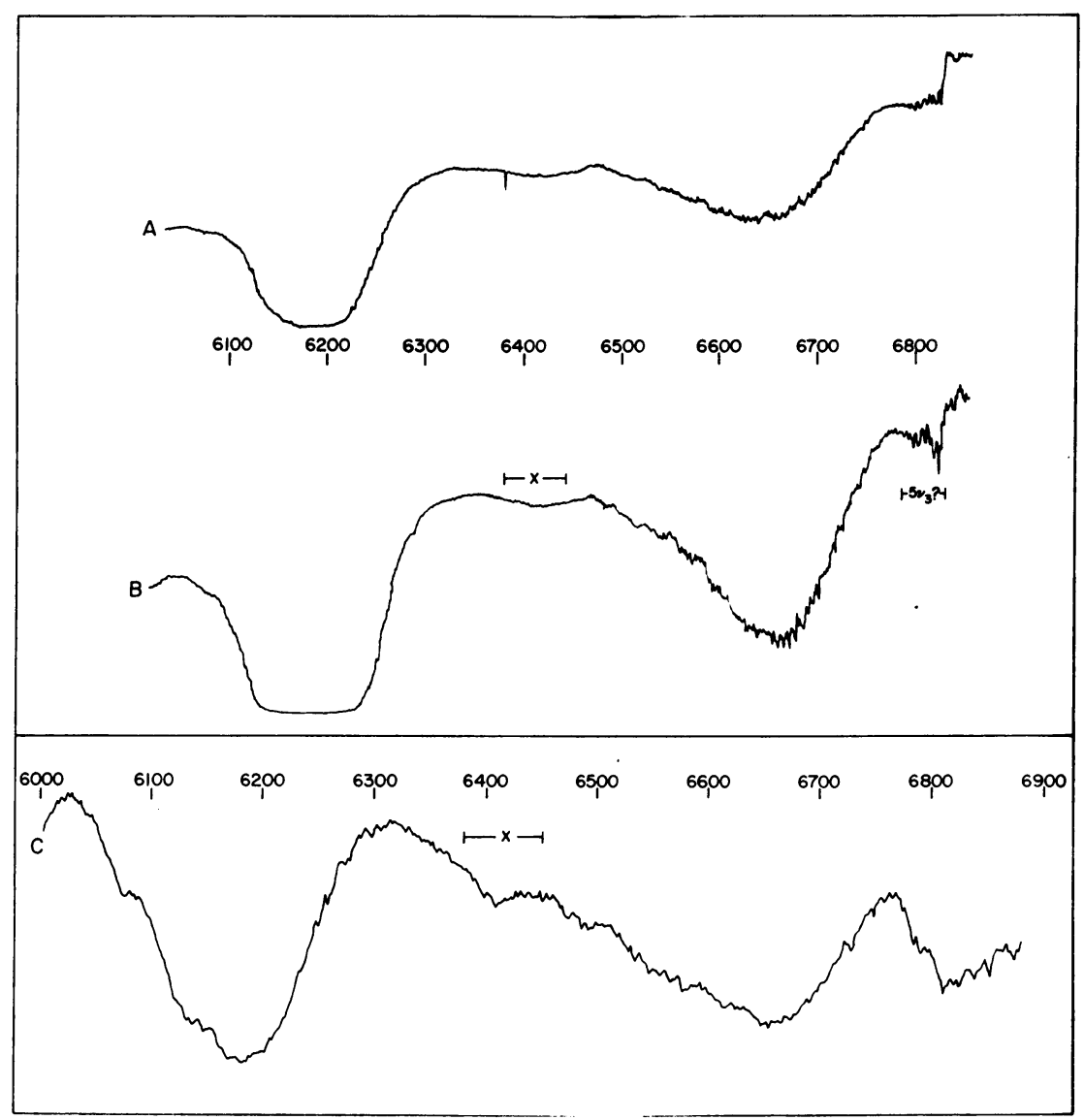

Fig. 1. Density tracings of spectrograms of methane - (A) $5.28 \mathrm{~km}$ amagat at $1.0 \mathrm{~atm}$, (B) $10.1 \mathrm{~km}$ amagat at $1.92 \mathrm{~atm}$. Original dispersion $4.56 \AA \mathrm{mm}^{-1}$, NRC, Ottawa, Canada. The sharp spike at $6420 \AA$ in (A) is a plate defect. (C) The spectrum of Uranus divided by that of the Moon. Original resolution, $2 \AA$, Tull coudé scanner at 2.7-m telescope, McDonald Observatory, U.S.A. (July, 1973).

\section{Abundances of Atmospheric Constituents}

\subsection{HYDROGEN}

A summary of recent abundance determinations from the $\mathrm{H}_{2}$ quadrupole lines in the spectrum of Uranus is given in Table IV of Encrenaz and Owen (1973). While there is still a considerable scatter in these results, convergence toward a value of $450 \pm 100 \mathrm{~km}$ am (for simple reflecting models) seems to be occurring. The first observations of quadrupole lines in the spectrum of Neptune have been reported at this conference by Trafton (1973), who found the equivalent widths to be similar to those for Uranus.

\subsection{Methane}

The original estimates of abundances by Kuiper (1952) and the revisions suggested by 
Owen (1967) are given below:

\begin{tabular}{lll} 
& Kuiper (1952) & Owen (1967) \\
\hline Uranus & $2.2 \mathrm{~km} \mathrm{am}$ & $3.5 \pm 1.2 \mathrm{~km} \mathrm{am}$ \\
Neptune & 3.7 & 6
\end{tabular}

The revisions seemed necessary to provide enough methane to account for the weak $7500 \AA$ lines referred to above. However, both sets of estimates were simply based on attempts to match observed intensities of the planetary absorptions with intensities in laboratory spectra of the gas. This a crude approach at best, since it ignores possible differences in pressure and temperature between the laboratory and the atmospheres of the planets.

If the hydrogen abundances mentioned above are correct, the effective pressure at the level of line formation will be of the order of $4 \mathrm{~atm}$ (Encrenaz and Owen, 1973). This means that weak methane bands in the planetary atmospheres will not be saturated and hence only the temperature difference is significant. By integrating the absorption over an entire band, we should be able to allow for this effect. We have therefore looked for weak bands in the planetary spectra at short wavelengths where we have some hope of identifying the true continuum.

In the region from 4500 to $5300 \AA$, we have found the following bands in spectra of both planets (Figure 2):

$\begin{array}{lll}5210 \AA & \mathrm{N} & \text { GBBM } \\ 5090 & & \text { GBBM } \\ 4950 & & \text { GBBM } \\ 4860 & & \text { GBBM } \\ 4590 & \mathrm{~N} & \end{array}$

This region was studied previously by Adel and Slipher (1934) who found all of the bands listed above except the one at $4950 \AA$ in the spectrum of Neptune. The two bands marked $\mathrm{N}$ were not found by them in the spectrum of Uranus, although they are definitely present (Figure 1). The bands marked GBBM were reported by Galkin et al. (1971) who only observed Uranus, and then only for $\lambda>4800 \AA$. We confirm their identification of the band at $4950 \AA$, the weakest member of this set.

In ratio spectra, we find that the 4860 and $4590 \AA$ bands are especially enhanced on Neptune compared with Uranus, while those at longer wavelengths (on our list) are more equal (Figure 2). But while Kuiper (1952) reported a ratio of 1.7 for the methane abundances in the atmospheres of the two planets, we find it to be closer to 1.25 , assuming that a ratio of the equivalent widths of these weak bands is in fact a measure of the abundance ratio. This result was obtained both from the McDonald observations shown in Figure 2 and from observations made by one of us (J.H.) at much lower resolution at the Smithsonian Astrophysical Observatory at Mt. Hopkins. The next step in this preliminary approach to the problem is to try to match the 


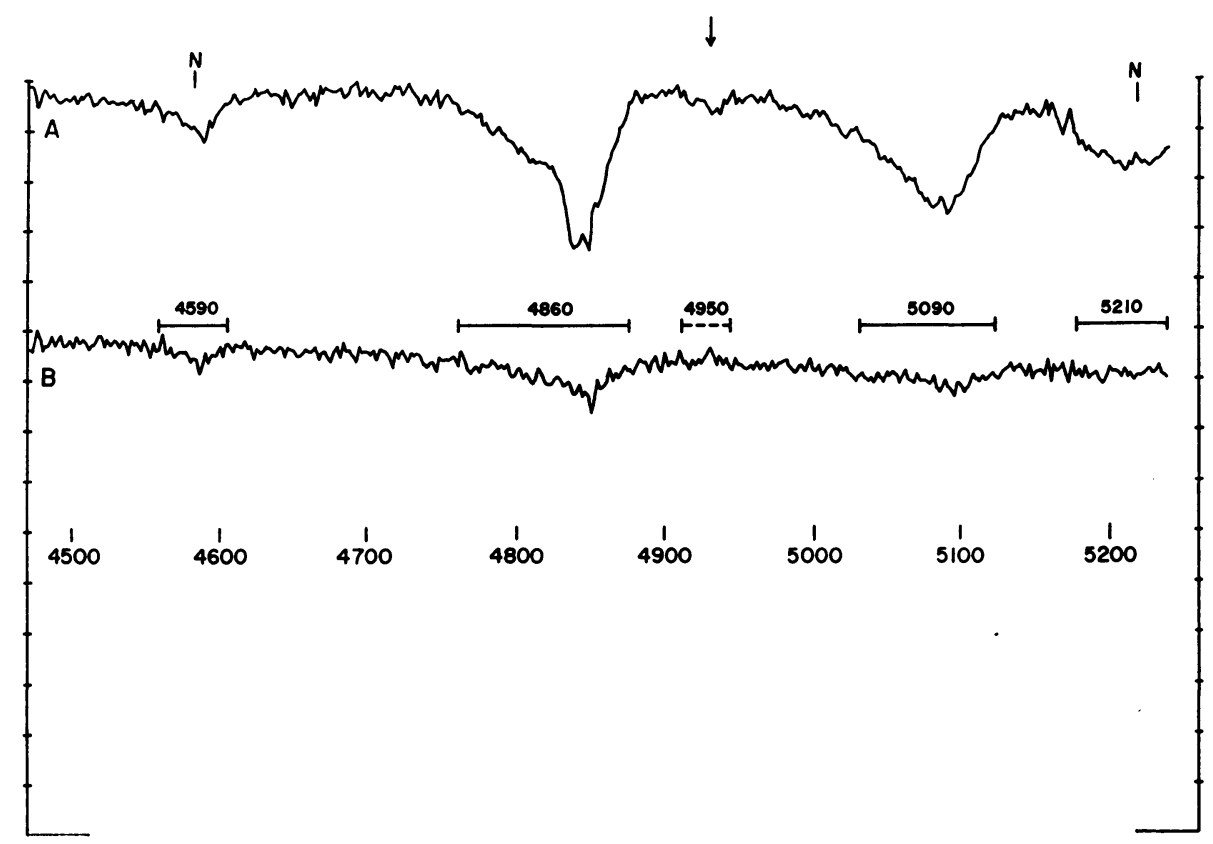

Fig. 2. (A) The spectrum of Uranus divided by that of the Moon. Arrow denotes location of $4950 \AA$ band, N refers to bands seen by Adel and Slipher (1934) in spectrum of Neptune only (see text). (B) The spectrum of Neptune divided by the spectrum of Uranus. Note residual absorptions. Both (A) and (B) obtained at $2 \AA$ resolution with Tull coudé scanner at $2.7-\mathrm{m}$ telescope, McDonald Observatory U.S.A. (July, 1973).

intensity of one of these weak bands in the planetary spectra with a suitable laboratory path length of methane.

We have examined spectra of 5.28 and $10.1 \mathrm{~km}$ of methane kindly obtained for us by Dr D. A. Ramsay at the NRC in Ottawa. The respective pressures were 1 and $1.9 \mathrm{~atm}$, always at room temperature (cf. Figure 1). We found traces of the $5970 \AA$ and $5760 \AA$ bands, but no hint of $5210 \AA$, which is considerably stronger than $4590 \AA$ or $4950 \AA$. One is thus led to the conclusion that the effective optical paths of methane in the atmospheres of these planets (including the air mass factor $\eta$ ) are much larger than $10 \mathrm{~km}$ am and may be in the range 50 to $100 \mathrm{~km}$ am. This in turn implies a value of $\mathrm{H} / \mathrm{C} \approx 40$, almost two orders of magnitude below the cosmic value exhibited by Jupiter and Saturn.

Clearly this estimate requires quantitative verification and we hope to be able to provide that in the near future through a study of the $6800 \AA$ band, tentatively identified as $5 v_{3}$ (cf. Figure 1), which seems to have a structure that is sufficiently simple to permit precise analysis (Owen, 1966). A recently obtained spectrogram of this band that illustrates its potential is reproduced in Figure 3 (see also Teifel and Kharitonova, 1970).

One other point should be mentioned. The fact that the weak methane absorptions in regions where the continuum is well-defined are definitely stronger on Neptune 


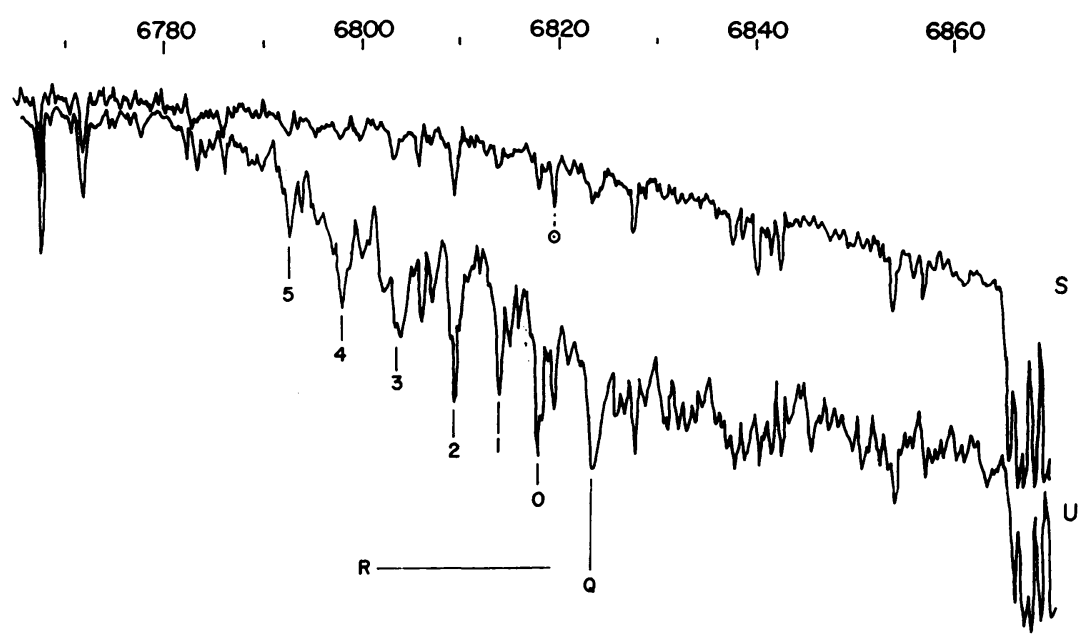

Fig. 3. Reproductions of spectrograms of Saturn (top, labeled ' $S$ ') and Uranus (bottom, labeled ' $U$ ') in the region of the putative $5 v_{3}$ band. The $J$ assignments are very tentative. Original dispersion $7 \AA$ $\mathrm{mm}^{-1}$, coudé spectrograph, 5-m telescope, Hale Observatories (March, 1972).

than on Uranus is an argument against the BMP model of a pure molecular semi-infinite atmosphere. It also suggests, counter to Morrison and Cruikshank (1973) and Belton and Spinrad(1973) that the atmospheric structures of these two planets are not identical.

\subsection{AmmoniA}

Danielson (private communication) has pointed out that in the case of a semi-infinite atmosphere on Uranus, a substantial number of photons should reach levels at which ammonia absorption will occur in the $6450 \AA$ region. Accordingly, we have examined the spectrum of Uranus in this region to see if any ammonia lines were present. We have not been able to find any evidence for ammonia, with a lower limit of $10-15 \mathrm{~m} \AA$ on the equivalent widths of the lines. Comparison with observations of this band in the spectrum of Jupiter (Mason, 1970) indicates that the total amount of ammonia in the line of sight must be less than $20 \%$ of the amount on Jupiter. This suggests $\mathrm{NH}_{3} \leqslant 2.5 \mathrm{~m}$ am in a one-way path in the atmosphere of Uranus. We have not carried out this kind of analysis for Neptune.

\subsection{OTHER GASES}

In their original identification of methane features in the spectra of these two planets, Adel and Slipher (1934) also examined the absorption spectra of long paths of ethylene $\left(\mathrm{C}_{2} \mathrm{H}_{4}\right)$ and ethane $\left(\mathrm{C}_{2} \mathrm{H}_{6}\right)$. They found no indication that either of these gases was present.

In the region we have investigated, the relevant absorptions Adel and Slipher reported are:

$$
\begin{array}{ll}
\mathrm{C}_{2} \mathrm{H}_{6} & 6320 \AA \\
\mathrm{C}_{2} \mathrm{H}_{4} & 6040 \AA .
\end{array}
$$


We must also report that we find no compelling evidence to suggest that either one of these gases is in the atmosphere of either planet. However, because of its interest in atmospheric photochemistry, we are undertaking a new investigation of the $\mathrm{C}_{2} \mathrm{H}_{6}$ spectrum in the laboratory and will reexamine the question of its existence in these atmospheres at a later date.

\section{Raman Effect}

Belton et al. (1973) have reported detection of the Raman effect in the spectrum of Uranus. Specifically, they have found an absorption near $4025 \AA$ which they attributed to the overlapping $\mathrm{H}$ and $\mathrm{K}$ lines shifted appropriately for the $S(0)$ and $S(1)$ rotational transitions in $\mathrm{H}_{2}$. The depression corresponds to an absorption of about $10 \%$, in accord with the prediction by Wallace (1972). They found the relative $S(0): S(1)$ Raman contributions to be of the order of $0.3: 0.7$. The published observations show no indication of the $K_{0}$ line, and only weak evidence for $\mathbf{H}_{1}$.

We have also looked for this effect in observations carried out with the Tull scanner at the McDonald Observatory in March and July of this year (1973). We studied both Uranus and Neptune, using Saturn's rings and the Moon to provide solar comparison spectra. Details will be presented elsewhere (Woodman et al., 1974), but we can report at this time that we find only weak evidence of an absorption at $4025 \AA$, with a central intensity no greater than $5 \%$. Additional study of the problem would seem to be advisable.

Even if Belton et al. (1973) are correct, however, the reader should not conclude that this observation supports the clear atmosphere model of BMP. In their discussion, Belton et al. (1973) point out that it is possible to have atmospheres with a tenuous haze or with a cloud layer that would also satisfy their observations.

\section{Conclusions}

The main results of this survey may be summarized as follows:

(1) The weight of present observational evidence is against the semi-infinite, puremolecular atmosphere originally proposed by BMP. It is not yet clear what the best alternative model is.

(2) The continuum in spectra of both planets is distorted by methane absorptions at wavelengths greater than $4700 \AA$.

(3) The absorption at $6420 \AA$ in spectra of both planets is mainly caused by methane, rather than the $\mathrm{S}_{4}(0)$ dipole absorption of $\mathrm{H}_{2}$.

(4) The abundance of methane is higher than had been thought, perhaps by as much as a factor 10, although this conclusion requires quantitative verification by studies of individual bands.

(5) The mixing ratio of methane to hydrogen is much higher in these atmospheres than in the atmospheres of Jupiter and Saturn, with values of $\mathrm{H} / \mathrm{C}$ below 100 a real possibility. Aside from its cosmogonical implications, this low ratio increases the 
chances of methane condensation in the upper atmospheres (Prinn and Lewis, 1973).

(6) The atmospheres of the two planets do not appear to be identical, despite many similarities.

\section{Acknowledgements}

Observations at the McDonald and Hale Observatories were made possible through the courtesy of Dr H. J. Smith and Dr H. W. Babcock, respectively. We are grateful for assistance in plate reductions from :Ms Carolyn Porco and for many helpful discussions with Dr Barry Lutz. The laboratory spectra which were critical to this work were generously obtained and made available by Dr D. A. Ramsay. The research was supported by NASA grants NGR-33-015-141, NGR-33-015-169, and NGR-33015-165.

\section{References}

Adel, A. and Slipher, V. M.: 1934, Phys. Rev. 46, 902.

Allen, C. W.: 1963, Astrophysical Quantities, Athlone Press, London, p. 143.

Belton, M. J. S., McElroy, M. B., and Price, M. J.: 1971, Astrophys. J. 164, 191.

Belton, M. J. S. and Price, M. J.: 1973, Astrophys. J. 179, 965.

Belton, M. J. S. and Spinrad, H.: 1973, Astrophys. J. 185, 363.

Belton, M. J. S., Wallace, L., and Price, M. J.: 1973, Astrophys. J. 184, L143.

Briggs, F. H.: 1973, Astrophys. J. 182, 999.

Danielson, R. E., Tomasko, M. G., and Savage, B. D.: 1972, Astrophys. J. $178,887$.

Encrenaz, T. and Owen, T.: 1973, Astron. Astrophys. 28, 119.

Galkin, L. S., Bugaenko, L. A., Bugaenko, O. I., and Morozhenko, A. V.: 1971, in C. Sagan, T. C. Owen, and H. J. Smith (eds.), 'Planetary Amospheres', IAU Symp. 40, 392.

Kovalevsky, J. and Link, F.: 1969, Astron. Astrophys. 2, 398.

Kuiper, G. P.: 1949, Astrophys. J. 109, 540.

Kuiper, G. P. (ed.): 1952, The Atmospheres of the Earth and Planets, Univ. of Chicago Press, Chicago, p.374.

Lutz, B. L. and Ramsay, D. A.: 1972, Astrophys. J. 176, 521.

Mason, H. P.: 1970, Astrophys. Space Sci. 7, 424.

Morrison, D. and Cruikshank, D. P.: 1973, Astrophys. J. 179, 333.

Owen, T.: 1966, Astrophys. J. 146, 611.

Owen, T.: 1967, Icarus 6, 108.

Owen, T.: 1969, Icarus 10, 355.

Owen, T., Lutz, B. L., Porco, C. S., and Woodman, J. H.: 1974, Astrophys. J. 189, 379.

Prinn, R. G. and Lewis, J. S.: 1973, Astrophys. J. 179, 333.

Savage, B. D. and Caldwell, J. C.: 1974, Astrophys. J. 187, 197.

Sinton, W. M.: 1972, Astrophys. J. 176, L131.

Smoluchowski, R.: 1973 in D. Gautier (ed.), Physics of Planets, Meudon: Groupe Planètes, p. 105.

Spinrad, H.: 1963, Astrophys. J. 138, 1242.

Teifel, V. G. and Kharitonova, G. A.: 1970, Astron. Zh. 13, 865.

Trafton, L.: 1974, this volume, p. 497.

Wallace, L.: 1972, Astrophys. J. 176, 249.

Westphal, J. A.: 1972, Bull. Am. Astron. Soc. 4, 361

Woodman, J. H., Owen, T., and Encrenaz, T.: 1974, in preparation.

\section{DISCUSSION}

Fox: Is it possible that the $6420 \AA$ feature is a combination of $\mathrm{H}_{2}$ and $\mathrm{CH}_{4}$ absorption?

Owen: It may be, but there are other arguments against the $\mathrm{H}_{2}$ interpretation; first, the absence of 
the $S(1)$ feature at $6470 \AA$, despite the measured continuum level at this wavelength, and second, the presence of structure at high resolution in the $6420 \AA$ region.

Williams: Have there been supporting laboratory studies at Uranus/Neptune temperatures?

Owen: There have only been a few studies at relatively short pathlengths at the appropriate temperatures. We are hoping to carry out better studies when a new, temperature-controlled cell is completed in Canada next year.

Trafton: What is the spectral resolution of the laboratory $\mathrm{CH}_{4}$ data at $6400 \AA$ ?

Owen: The dispersion is $4.5 \AA \mathrm{mm}^{-1}$. The planetary spectrum has a resolution element of $4 \AA$. 